\title{
A SYSTEM OF VAN DER POL TYPE UNDER NONLINEAR PARAMETRIC EXCITATION
}

\author{
NGUYen Van Dinh - Tran Kim Chi \\ Institute of Mechanics, Hanoi Vietnam
}

In [1], the interaction between self-excited oscillation and parametric one in a system of Van der Pol type subjected to nonlinear restoring force harmonically depending on time has been studied. There, the parametric excitation is rather intense (in comparaison with the self-excitation) while its nonlinearity is relatively weak; consequently, the resonance curves obtained are similar to those of the well-known "pure" parametrically excited system. Below a system of same category will be considered, assuming that the intensities of the two excitations are of same order and as in $[2$, chapter $4, \S 4]$ for a generalized Mathieu system - the time-dependent nonlinearity is strong enough. With these assumptions, the form of the resonance curve is various. In order to reveal all possible forms of the resonance curve, the so-called critical stationary oscillations will be used $[3,4]$.

\section{System under consideration}

Let us consider a quasi-linear oscillating system described by the differential equation:

$$
\ddot{x}+\omega^{2} x=\varepsilon\left\{\Delta x+h\left(1-k x^{2}\right) \dot{x}+2 \mu\left(x-p x^{2}+q x^{3}\right) \cos n \omega t\right\}
$$

where: $x$ is an oscillatory variable; overdots denote differentiation with respect to time $t ; \varepsilon>0$ is a small parameter; $(h>0 ; k>0)$ and $(2 \mu>0 ; p ; q)$ are coefficients respectively characterizing the self-excitation and the parametric one; $n \omega(n=1,2,3)$ is the exciting frequency; $\varepsilon \Delta=\omega^{2}-1$ is the detuning parameter ( 1 - own frequency).

Introducing slowly varying amplitude $a$ and dephase $\theta$ by means of formulas:

$$
x=a \cos \psi, \quad \dot{x}=-\omega a \sin \psi, \quad \psi=\omega t+\theta
$$

and applying the asymptotic method, we obtain:

$$
\begin{gathered}
\dot{a}=\frac{-\varepsilon a}{2 \omega} f_{n}=\frac{-\varepsilon a}{2 \omega}\left\{h \omega\left(\frac{1}{4} k a^{2}-1\right)-F_{n}\right\} \\
a \dot{\theta}=\frac{-\varepsilon a}{2 \omega} g_{n}=\frac{-\varepsilon a}{2 \omega}\left\{\Delta-G_{n}\right\} \quad(n=1,2,3)
\end{gathered}
$$

where

$$
\begin{aligned}
& F_{1}=\frac{1}{2} \mu p a \sin 2 \theta, \quad F_{2}=\mu\left(\frac{1}{2} q a^{2}-1\right) \sin 2 \theta, \quad F_{3}=\frac{1}{2} \mu p a \sin 3 \theta, \\
& G_{1}=\frac{3}{2} \mu p a \cos 2 \theta, \quad G_{2}=\mu\left(q a^{2}-1\right) \cos 2 \theta, \quad G_{3}=\frac{1}{2} \mu p a \cos 3 \theta .
\end{aligned}
$$

The equations for determinating stationary oscillations are:

$$
f_{n}=0, \quad g_{n}=0, \quad(n=1,2,3)
$$


2. The cases $n=1 ; 3$

In these cases, the resonance curves only consist of the ordinary parts given respectively by:

$$
\begin{aligned}
& \text { - for } n=1: W\left(\Delta, a^{2}\right)=h^{2} \omega^{2}\left(\frac{1}{4} h a^{2}-1\right)^{2}+\frac{\Delta^{2}}{9}-\frac{1}{4} \mu^{2} p^{2} a^{2}=0 \\
& \text { - for } n=3: W\left(\Delta, a^{2}\right)=h^{2} \omega^{2}\left(\frac{1}{4} k a^{2}-1\right)^{2}+\Delta^{2}-\frac{1}{4} \mu^{2} p^{2} a^{2}=0
\end{aligned}
$$

Since $h$ is assumed to be small $(h \ll 1)$ the resonance curves do not intersect the abscissa axis $\Delta$ (this means that the effect of the parametric excitation is relatively weak). Moreover, the resonance curves always pass through the ends of a segment-denoted by $J_{0}$ - parallel to the axis $\Delta$, respectively given by:

$$
\begin{aligned}
& \text { - for } n=1:-3 \mu p / \sqrt{k} \leq \Delta \leq 3 \mu p / \sqrt{k} ; \quad a^{2}=a_{0}^{2}=\frac{4}{k} \\
& \text { - for } n=3:-\mu p / \sqrt{k} \leq \Delta \leq \mu p / \sqrt{k} ; \quad a^{2}=a_{0}^{2}=\frac{4}{k}
\end{aligned}
$$

$a_{0}^{2}=\frac{4}{k}$ is the amplitude of the "pure" self-excited oscillation $(\mu=0)$; so, $J_{0}$ represents the "level" of the oscillation in the "original" Van der Pol system (this level is related to the negative friction $k$-the ratio between the negative friction and the positive one). As an illustration, let us choose $n=3 ; \mu=0.05 ; p=1 ; k=4\left(a_{0}^{2}=1\right)$. The resonance curves (1) (2) shown in Fig. 1 have been drawn for $h=0.05 ; 0.1$, respectively. We see that the resonance curves are of form "oval" and "envelop" the segment $J_{0}$. Increasing $h$ (the positive friction - the intensity of the self-excitation), the resonance curves become narrower and "tend" to $J_{0}$ (this means that when $h$ is large enough, the amplitude of the "mixed" oscillation is near that of the pure self-excited one). Evidently, if $h$ is too

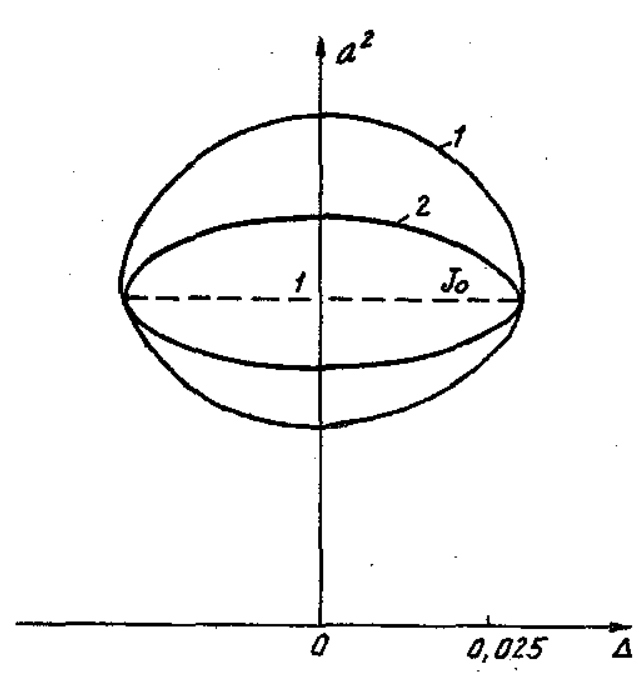

Fig. 1 small or if $a_{0}^{2}=\frac{4}{k}$ is too large ( $k$ small the segment $J_{0}$ goes up to infinity), the resonance curves take the form "parabola" (the half-lower part of the mentioned "oval")

\section{The cases $n=2$. Ordinary and critical stationary oscillations}

In this case, the equations (1.4) are:

$$
\begin{aligned}
\mu\left(\frac{1}{2} q a^{2}-1\right) \sin 2 \theta & =h \omega\left(\frac{1}{4} k a^{2}-1\right), \\
\mu\left(q a^{2}-1\right) \cos 2 \theta & =\Delta .
\end{aligned}
$$

In the ordinary region where:

$$
\left(\frac{1}{2} q a^{2}-1\right)\left(q a^{2}-1\right) \neq 0
$$


the ordinary part of the resonance curve is given by:

$$
W\left(\Delta, a^{2}\right)=\frac{h^{2} \omega^{2}\left(\frac{1}{4} k a^{2}-1\right)^{2}}{\mu^{2}\left(\frac{1}{2} q a^{2}-1\right)^{2}}+\frac{\Delta^{2}}{\mu^{2}\left(q a^{2}-1\right)^{2}}-1=0 .
$$

The critical region in characterized by the equality:

$$
\left(\frac{1}{2} q a^{2}-1\right)\left(q a^{2}-1\right)=0 .
$$

It consists of two straight lines-denoted by (I) (II) respectively - parallel to the abscissa $\Delta$ :

$$
\text { (I) : } \quad a^{2}=a_{1}^{2}=\frac{1}{q} ; \quad \text { (II) : } \quad a^{2}=a_{2}^{2}=\frac{2}{q} \text {. }
$$

If $q>0$ and large enough, the two critical lines (I) (II) are present in the acceptable resonance domain ( $a^{2}$ not too large). Along (I), the equations (3.1) become:

$$
-\frac{1}{2} \mu \sin 2 \theta=h \omega\left(\frac{h}{4 q}-1\right), \quad 0 \cos 2 \theta=\Delta .
$$

From these equations, it is easy to deduce that the compatible point $I_{*}$ of coordinates

$$
\Delta_{*}=0, \quad a_{*}^{2}=a_{1}^{2}=\frac{1}{q}
$$

is a critical representing point if

$$
h \leq h_{*}=\frac{\mu}{2}\left|\frac{k}{4 q}-1\right| \text {. }
$$

Analogously, when $k=2 q$, the critical line (II) contains a critical segment - denoted by $J_{1} J_{2}$ determined by:

$$
-\mu \leq \Delta \leq \mu ; \quad a^{2}=a_{2}^{2}=\frac{2}{q} .
$$

The "whole" resonance curve can be determined by the relationship

$$
\begin{aligned}
W\left(\Delta, a^{2}\right)= & h^{2} \omega^{2}\left(\frac{1}{4} k a^{2}-1\right)^{2}\left(q a^{2}-1\right)^{2}+\left(\frac{1}{2} q a^{2}-1\right)^{2} \Delta^{2} \\
& -\mu^{2}\left(\frac{1}{2} q a^{2}-1\right)^{2}\left(q a^{2}-1\right)^{2}=0,
\end{aligned}
$$

on condition that must be rejected the point $I_{*}$ when $h>h_{*}$ and also those points of (II) do not satisfying the inequalities (3.9), if $k=2 q$.

If $h<h_{*}, I_{*}$ is a nodal point; if $h=h_{*}, I_{*}$ is either a returning point or a degenerated nodal one (with a double tangent); if $h>h_{*}, I_{*}$ becomes an isolated point, it does not belong to the resonance curve.

As in $\$ 2$, the resonance curve possesses some interesting properties:

- For small $h: h \leq h_{1}=\left(2-2 \sqrt{1-\mu^{2}}\right)^{1 / 2}(a$ little larger than $\mu)$, the resonance curve intersects $\Delta$

- The resonance curve always passes through the two ends of the segment $J_{0}$ which is now given by

$$
-\mu\left|\frac{4 q}{k}-1\right| \leq \Delta \leq \mu\left|\frac{4 q}{k}-1\right| ; \quad a^{2}=a_{0}^{2}=\frac{4}{k} .
$$


If $k=2 q, y_{0}$ becomes $J_{3} J_{2} ;$ if $4 q=k, y_{0}$ is reduced to the point $I_{*}\left(\Delta_{*}=0, a_{0}^{2}=\frac{4}{k}=\frac{1}{q}=a_{*}^{2}\right)$.

4. The case $n=2$. Various forms of the resonance curve

The form of the resonance curve essentially depends on:

- the rate between the intensity $h$ of the self-excitation (the positive friction) and the intensity $\mu$ of the linear parametric one.

- the relative distribution between the segment $J_{0}$ and the two critical lines (I) (II). Let us recall that $J_{0}$ represents the level of the pure self-excited oscillation and it is determined by $k$-the negative friction. As to (I) (II), they are related to $q$-the ratio between the cubic and the linear coefficients of the time dependent restoring force.

We shall fix the parametric excitation: $\mu=$ $0.05 ; q=2$; so: $a_{1}^{2}=\frac{1}{2} ; a_{2}^{2}=1 ; h_{1} \approx 0.050016>$ $\mu$. Then for each chosen value $k$, the resonance curves will be drawn for different values $h$.

1. The case $k<4$ (but not too small): $a_{0}^{2}=$ $\frac{4}{k}>a_{2}^{2}=1, J_{0}$ lies above (II). Let us choose $k=3$; so: $a_{0}^{2}=\frac{4}{3}>1 ; h_{*}=0.04<\mu<h_{1}$. The resonance curves (1) (2) (3) (4) shown in Fig. 2 have been drawn for $h=0.025 ; 0.04 ; 0.045 ; 0.055$ respectively. The resonance curve consists of two branches, separated by the critical line (II). The upper branch $C^{\prime \prime}$ always passes through the two ends of the segment $J_{0}\left(\frac{-0.25}{3} \leq \Delta \leq \frac{0.25}{3} ; a^{2}=\right.$ $\left.\varsigma_{0}^{2}=\frac{4}{3}\right)$. As $h$ increases, $C^{\prime \prime}$ becomes narrower,

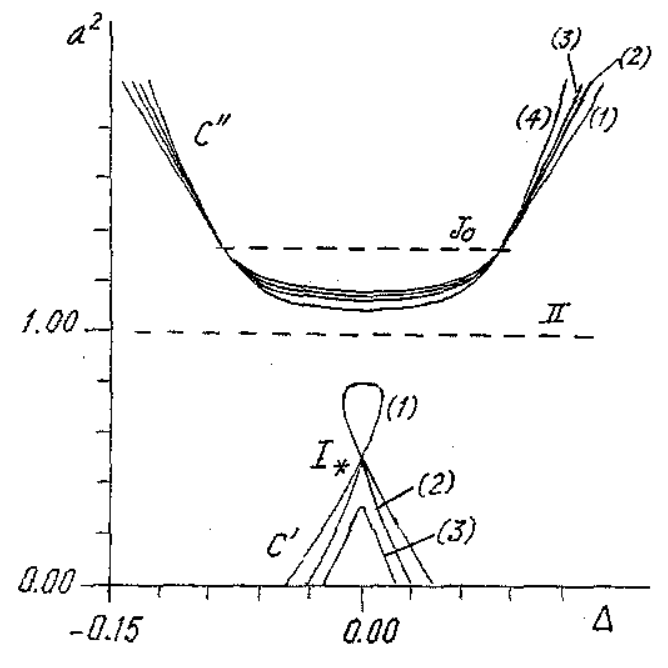

Fig. 2

it will envelop $J_{0}$ then finally tends to $J_{0}$. For small $h$, the lower branch $C^{\prime}$ intersects $\Delta$ and has a rodal point $I_{*}$. As $h$ reaches the value $h_{*}, I_{*}$ becomes a returning point; then, it changes into an isolated point when $h>h_{4}$. The lower branch $C^{\prime}$ continues to cut $\Delta$ until $h=h_{1}$ at which, it is transformed into a (very small) "oval" tangent to $\Delta$. Increasing further $h$, the "oval" $C^{\prime}$ leaves $\Delta$, it becomes narrower then finally disappears.

2. The case $k=4$ - This is the particular case $k=2 q$. The segment $J_{0}$ becomes the critical segment $J_{1} J_{2}\left(-0.05 \leq \Delta \leq 0.05 ; a^{2}=a_{0}^{2}=a_{*}^{2}=1\right), h_{*}=0.05=\mu<h_{1}$. In Figs 3,4 the resonance curves (1) (2) (3) (4) (5) correspond to $h=0.04 ; 0.05 ; 0.050005 ; 0.050010 ; 0.050020$ respectively. For every value $h$, the segment $J_{1} J_{2}$ is the critical segment. If $h<0.05=h_{*}$, the reson ance curve intersects $\Delta$ and has a nodal point $I_{*}$. As $h=h_{*}=0.05, I_{*}$ becomes a degenerated nodal point. For $h>h_{*}$, the upper and lower branches $C^{\prime \prime}$ and $C^{t}$ appear. Increasing further $h$, the upper branch $C^{\prime \prime}$ moves up, the lower one $C^{\prime}$ moves down, changes into an "oval" then finally disappears

3. The case $4<k<8: a_{1}^{2}=\frac{1}{2}<a_{0}^{2}=\frac{4}{k}<1=a_{2}^{2}, J_{0}$ lies between two critical lines (I) and (II). Let us choose $k=5$; so: $a_{0}^{2}=\frac{4}{5} ; h_{*}=\frac{0.2}{3}>h_{1}>\mu$. The resonance curves (1) (2) (3) (4) (5) shown in Fig. 5 have been drawn for $h=0.0300 ; 0.0500 ; 0.0550 ; 0.0666 ; 0.0800$ respectively. We also obtain a resonance curve with two branches, separated by the critical line (II). As $h$ increases, 
the upper branche $C^{\prime \prime}$ moves up. For small $h, C^{\prime}$ intersects $\Delta$ and has a nodal point $I_{*}$. $C^{\prime}$ intersects $\Delta$ until $h=h_{1}$ at which it becomes to take the form of an eight. Increasing further $h$, the lower "loop" of the eight becomes narrower then disappears, $I_{*}$ changes into a returning point then an isolated one, $C^{\prime}$ is finally transformed into an "oval", enveloping $J_{0}$ and tends to $J_{0}$.

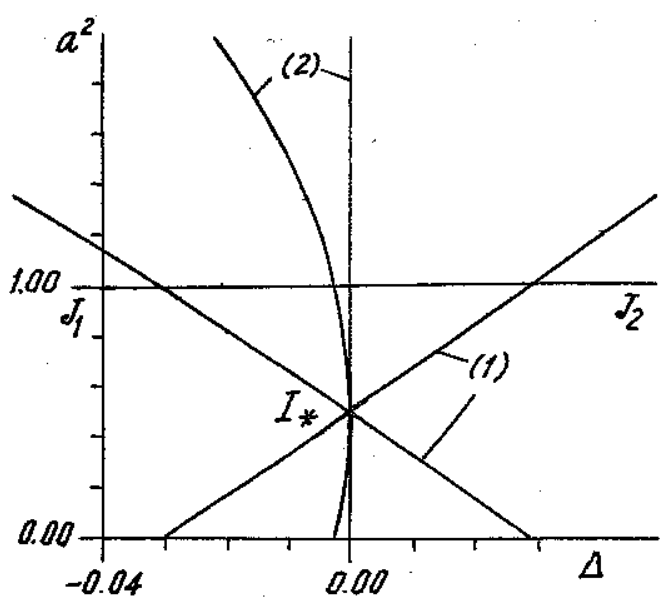

Fig. $\$$

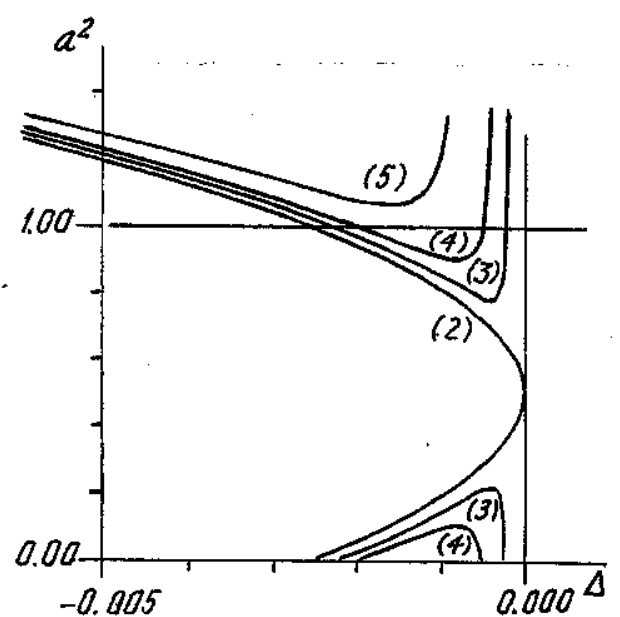

Fig. 4

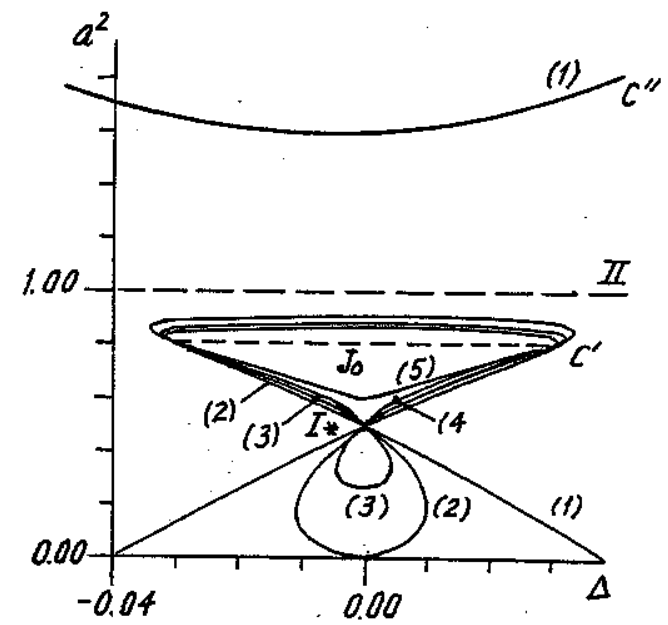

Fig. 5

4. The case $k=8 a_{0}^{2}=\frac{4}{k}=\frac{1}{2}=a_{1}^{2}, J_{0}$ is reduced to $I_{*}, h_{*}=\infty$. In Fig. 6 , the resonance curves (1) (2) (3) correspond to $h=0.04 ; 0.05 ; 0.06$ respectively. The critical point "persists" and the eight is the "final" form of the resonance curve.

5. The case $k>8: a_{0}^{2}<\frac{1}{2}=a_{1}^{2}, J_{0}$ lies under the critical line (I). Let us choose $k=12$; so: $a_{0}^{2}=\frac{1}{3}<\frac{1}{2} ; h_{*}=0.05<h_{1}$. The resonance curves (1) (2) (3) (4) shown in Fig. 7 correspond to $h=0.02 ; 0.03 ; 0.05 ; 0.08$ respectively 


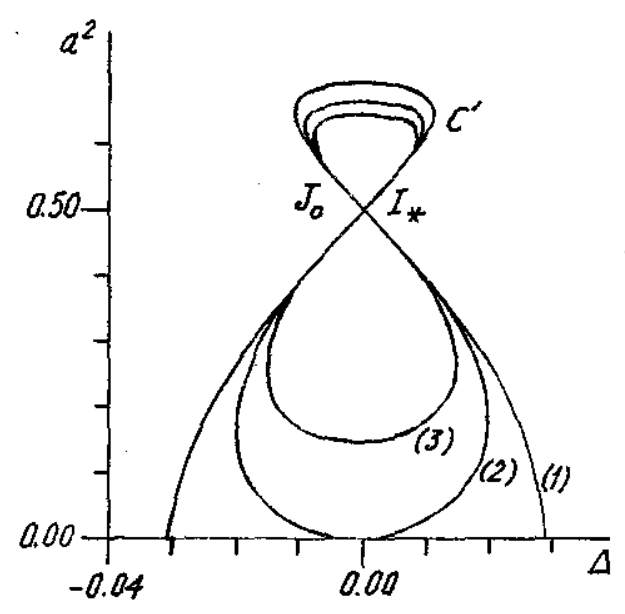

Fig. 6

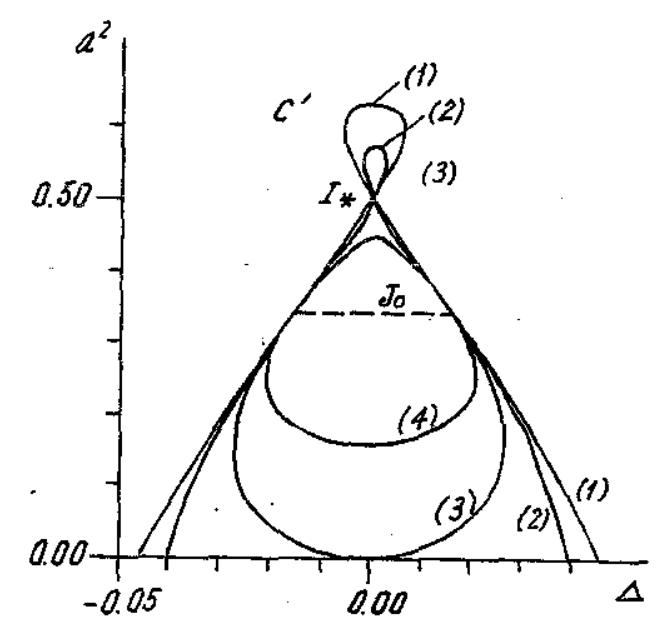

Fig. 7

\section{Some supplementary remarks}

Our main purpose is to reveal all the possible forms of the resonance curve. On other features of the system considered, we give some supplementary remarks

1. The time-dependent nonlinearity is assumed to be strong enough; so, $q$ is large and the critical part $\left(I_{*}\right.$ and $\left.J_{1} J_{2}\right)$ of the resonance curve is located in the acceptable ( $a^{2}$ is not too large) resonance domain. If the mentioned nonlinearity is weak ( $q$ small) or of soft kind $(q<0)$, the critical part "goes out off" the resonance domain. In this case, the resonance curve is relatively simple. As an illustration, in Fig. 8, for $\mu=0.05\left(h_{1} \approx 0.050016\right), k=4\left(a_{0}^{2}=1\right), q=0.2\left(a_{1}^{2}=5\right.$; $a_{2}^{2}=10$ ), the resonance curves (1) (2) (3) correspond to $h=0.04 ; 0.05 ; 0.06$ respectively.

2. The system (1.1) does not contain "free" nonlinear terms. If these terms are present and small, the results obtained above can be applied with light modifications. For example, in the case $n=3$ with the presence of free cubic term $\left(-\varepsilon \gamma x^{3}\right)$, we have:

- the equations of stationary oscillations:

$$
\begin{aligned}
\mu\left(\frac{1}{2} g a^{2}-1\right) \sin 2 \theta & =h \omega\left(\frac{1}{4} k a^{2}-1\right) \\
\mu\left(g a^{2}-1\right) \cos 2 \theta & =\left(\Delta-\frac{3 \gamma}{4} a^{2}\right)
\end{aligned}
$$

- the "invariants":

$$
\left(J_{0}\right): a_{0}^{2}=\frac{4}{k}, \quad \text { (I) }: a_{1}^{2}=\frac{1}{q}, \quad \text { (II) }: a_{2}^{2}=\frac{2}{q}, \quad h_{1}=\left(2-2 \sqrt{1-\mu^{2}}\right)^{1 / 2}
$$

- the coordinates of $I_{*}$ :

$$
\Delta_{*}=\frac{3 \gamma}{4} a_{1}^{2}=\frac{3 \gamma}{4 q}, \quad a_{*}^{2}=a_{1}^{2}=\frac{1}{q}
$$

and

$$
h_{*}=\frac{\mu}{2}\left|\frac{k}{4 q}-1\right| \sqrt{1+\frac{3 \gamma}{4}}
$$


- the segment $J_{0}$ :

$$
\frac{3 \gamma}{4} a_{0}^{2}-\mu\left|q a_{0}^{2}-1\right| \leq \Delta \leq \frac{3 \gamma}{4} a_{0}^{2}+\mu\left|q a_{0}^{2}-1\right|, \quad a_{0}^{2}=\frac{4}{k}
$$

which becomes the critical segment $J_{1} J_{2}$ when $k=2 q$ :

$$
\frac{3 \gamma}{2 q}-\mu \leq \Delta \leq \frac{3 \gamma}{2 q}+\mu, \quad a^{2}=a_{2}^{2}=\frac{2}{q}
$$

- the relationship (3.10):

$$
\begin{aligned}
W(\Delta, a)= & h^{2} \omega^{2}\left(\frac{1}{4} k a^{2}-1\right)^{2}\left(q a^{2}-1\right)^{2}+\left(\Delta-\frac{3 \gamma}{4} a^{2}\right)^{2}\left(\frac{1}{2} q a^{2}-1\right)^{2} \\
& -\mu^{2}\left(\frac{1}{2} q a^{2}-1\right)^{2}\left(q a^{2}-1\right)^{2}=0
\end{aligned}
$$

The resonance curves (1) (2) (3) shown in Fig. 9 have been drawn for $\mu=0.05 ; q=2 ; k=0.055$; $k=5$ and $\gamma=0.08 ; 0.12 ; 0.16$ respectively. The resonance curves lean to the right, it is the main effect of the positive free cubic nonlinearity

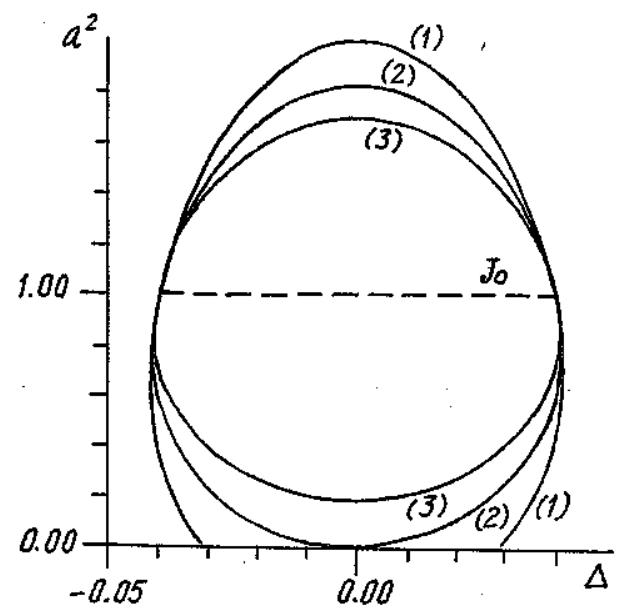

Fig. 8

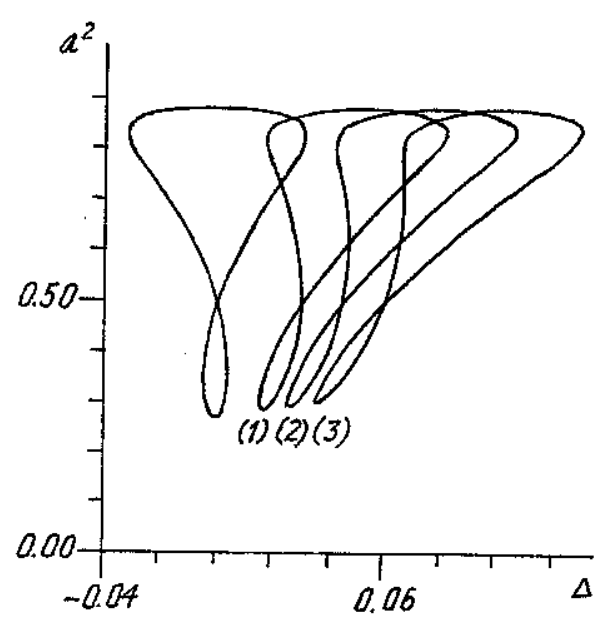

Fig. 9

3. The stability study is based on the variational equations and the two stability conditions are:

$$
a^{2}>a_{1}^{2}=\frac{1}{q}, \quad \frac{1}{\left(\frac{1}{2} q a^{2}-1\right)\left(q a^{2}-1\right)} \frac{\partial W}{\partial a^{2}}>0 .
$$

\section{Conclusion}

The interaction between self-excited oscillation and parametric one in a system of Van der Pol type subjected to strong time-dependent nonlinearity is examined. The form of the resonance curve is various and depends on the intensities of the two excitations as well as on the nonlinearities.

This publication is completed with financial support from the National Basic Research Programme in Natural Sciences. 


\section{REFERENCES}

1. Yano S. Analytic research on dynamic phenomena of parametrically and self-excited mechanical systems. Ingenieur Archiv, 57 (1987), 51-60.

2. Mitropolski Yu. A., Nguyen Van Dao. Applied asymptotic methods in nonlinear oscillations. Hanoi 1994.

3. Nguyen Van Dinh. Stationary oscillations in degenerated case. Journal of Mechanics, NCNST of Vietnam, T. XVIII, No 2, 1996, 13-19.

4. Nguyen Van Dinh. Stability of the critical stationary oscillations. Journal of Mechanics, NCNST of Vietnam, T. XIX, No 2, 1997, 15-20.

Received September 16, 1997

\section{Hệ VAN DER POL CHỊU KfCH ĐộNG THÔNG SỐ PHI TUYẾN}

Khảo sát tương tác giữa dao động thông số và tự chấn trong một hệ loại Van der Pol chịu kích động thông số phi tuyến. Các dao động dừng tới hạn tồn tại. Các đường cộng hường rất đa dạng và tùy thuộc cường độ các kích động cû̉ng như mức độ phi tuyến. 